\title{
Combined effect of toe out gait and high tibial osteotomy on knee adduction moment in patients with varus knee deformity
}

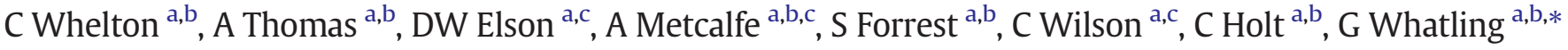 \\ a Arthritis Research UK Biomechanics and Bioengineering Centre, Cardiff University, Cardiff, UK \\ ${ }^{\mathrm{b}}$ Cardiff School of Engineering, Trevithick Building, Cardiff, UK \\ c Cardiff and Vale Orthopaedic Centre, University Hospital of Wales, Cardiff, UK
}

\section{A R T I C L E I N F O}

Article history:

Received 12 August 2015

Accepted 13 February 2017

\begin{abstract}
A B S T R A C T
Background: Gait adaptations, including toe out gait, have been proposed as treatments for knee osteoarthritis. The clinical application of toe out gait, however, is unclear.

This study aims to identify the changes in Knee adduction moment in varus knee deformity assessing toe out gait as an alternative to high tibial osteotomy, and if any change in dynamic loading persists post operatively, when anatomical alignment is restored.

Methods: Three-dimensional motion analysis was performed on 17 patients with medial compartment knee osteoarthritis and varus deformity prior to undergoing high tibial osteotomy, 13 patients were assessed post-operatively, and results compared to 13 healthy controls.

Findings: Pre-operatively, there was no significant difference between natural and toe out gait for measures of knee adduction moment. Post high tibial osteotomy, first $(2.70$ to $1.51 \% \mathrm{BW} \cdot \mathrm{h})$ and second peak ( 2.28 to $1.21 \% \mathrm{BW} \cdot \mathrm{h}$ ) knee adduction moment were significantly reduced, as was knee adduction angular impulse $(1.00$ to $0.52 \% \mathrm{BW} \cdot \mathrm{h} \cdot \mathrm{s})$, to a healthy level. Adopting toe out gait post-operatively reduced the second peak further to a level below that of healthy controls.

Interpretation: Increasing the foot progression angle from $20^{\circ}$ (natural) to $30^{\circ}$ in isolation did not significantly alter the knee adduction moment or angular impulse. This suggests that adopting a toe out gait, in isolation, in an already high natural foot progression angle, is not of benefit. Adopting toe out gait post-operatively, however, resulted in a further reduction in the second peak to below that of the healthy control cohort, however, this may increase lateral compartment load.
\end{abstract}

(c) 2017 Published by Elsevier Ltd.

\section{Introduction}

The medial compartment of the knee transmits the majority of load (Chang et al., 2007), and medial compartment overload is a risk factor for osteoarthritis progression (Miyazaki et al., 2002). The external knee adduction moment (KAM) has been studied as a surrogate measure of medial compartment load (Hunt and Bennell, 2011; Mundermann et al., 2005; Rutherford et al., 2008; Schache et al., 2008; Simic et al., 2011; Thorp et al., 2006; Thorp et al., 2007; Walter et al., 2010; Zhao et al., 2007) and it is accepted that medial loading is predominantly reflected by the KAM (Simic et al., 2011; Thorp et al., 2006; Walter et al., 2010; Zhao et al., 2007). A high KAM has been associated with disease progression (Bennell et al., 2011; Miyazaki et al., 2002), and lowering KAM has therefore been targeted to modify OA

\footnotetext{
* Corresponding author at: School of Engineering Cardiff University Queens Buildings, The Parade Cardiff CF24 3AA Wales UK

E-mail address: Whatlinggm@cardiff.ac.uk (G. Whatling).
}

progression (Caldwell et al., 2013). Methods to offload the medial compartment include gait adaptation, mechanical walking aids and surgical re-alignment.

Alteration of the foot progression angle (FPA), notably adopting a toe out gait (TOG), is one such gait adaptation. TOG has been shown to alter the two peaks of the KAM differently, either not affecting (Andrews et al., 1996; Caldwell et al., 2013; Guo et al., 2007; Lynn and Costigan, 2008; Schache et al., 2008; Shull et al., 2013) or increasing (Bennell et al., 2011; Hurwitz et al., 2002; Lin et al., 2001; Lynn et al., 2008; van den Noort et al., 2013) the first peak and reducing the second peak KAM (Caldwell et al., 2013; Guo et al., 2007; Jenkyn et al., 2008; Lin et al., 2001; Lynn and Costigan, 2008; Lynn et al., 2008; Reinbolt et al., 2008; Schache et al., 2008; van den Noort et al., 2013). A reduction is observed for the second peak KAM by adopting TOG but clinical benefit has yet to be established (Hunt and Takacs, 2014; Simic et al., 2011). Knee Adduction Angular impulse has been studied as a dynamic representation of medial knee load, and has found to be predictive of cartilage loss over 12 months (Bennell et al., 2011) and is more sensitive than peak KAM at distinguishing disease severity (Kean et al., 2012). 
High tibial osteotomy (HTO) is a surgical procedure that aims to realign the lower limb and has been shown to reduce both peaks of the KAM (Amis, 2013), with some degree of longevity (Bhatnagar and Jenkyn, 2010; Weidenhielm et al., 1992). The alteration in KAM is also associated with improved pain and function scores (Birmingham et al., 2009).

This study aims to identify differences in KAM between TOG and HTO. These treatments aim to offload the medial compartment with dynamic or anatomical compensation of varus knee deformity. This study aims to compare these adaptations, and to identify if any benefit in KAM can be attained with TOG after HTO, in order to compare the benefits of this gait adaptation after the deformity is corrected. The aim is to assess TOG as an alternative to surgery or an addition to surgery by its effect on KAM, which has not previously been reported.

\section{Methods}

\subsection{Study}

This was a controlled cohort study. Healthy volunteers participated as control subjects and were compared to a patient cohort who underwent HTO. Healthy volunteers were recruited, with no current knee symptoms and no previous knee surgery. Patients were diagnosed with medial compartment knee OA on the basis of history, clinical examination and plain radiographs were graded (Kellgren and Lawrence, 1957) for disease severity. Varus deformity was confirmed on full leg weight bearing radiographs. Subsequently patients scheduled to undergo HTO were invited to participate in this study. The study was part of ongoing research associated with the Arthritis Research UK, Biomechanics and Bioengineering Centre. Local research ethics committee approval was obtained.

\subsection{Motion analysis}

Motion analysis was performed using a three dimensional optoelectronic motion analysis system (Qualisys, Gothenburg, Sweden) using nine Oqus optoelectronic digital infrared cameras capturing at $120 \mathrm{~Hz}$. These were synchronised with ground force plates capturing at $1080 \mathrm{~Hz}$ (Bertec, Columbus, Ohio, USA).

Passive reflective markers were attached to the subjects in a modification of the Cleveland clinic configuration, with two additional markers on the hind foot segment of each foot as described by Reinbolt et al. (2008) resulting in a total of 37 markers (Reinbolt et al., 2008).

Participants walked along the walkway at self-selected speeds with variable starting points to ensure satisfactory striking of the force plates. After a period of practice to allow familiarity, the participants performed 6 walking trials with satisfactory force plate strikes with their natural foot progression angle. A verbal and visual demonstration of TOG was provided and participants were then asked to walk with their feet turned outwards, to a comfortable degree. The angle achieved was therefore self-selected but within the boundaries of what would be achievable and tolerated by patients in clinical practice. The pre- and post-operative patient groups, then performed 6 satisfactory walking trials with TOG.

Healthy volunteers in the control group visited the motion analysis laboratory once, and patients attended pre-operatively and were invited to return post operatively.

Data was processed using Visual 3D (C-motion, Inc. Maryland, USA) to compute kinetic and kinematic data, where a bespoke model and analysis pipeline was applied to each participants static measurement and dynamic trials. A Butterworth fourth order filter was applied to the raw marker coordinate data with a cut-off frequency of $6 \mathrm{~Hz}$, allowing the capture of lower frequency trajectory related data, and removing higher frequency data (digitisation noise). Joint rotations were calculated using Cardan sequence XYZ. A virtual foot was constructed to create a coordinate system parallel to the floor, in order to maintain clinical relevance. The foot progression angle was defined as the angle between the foot vector and the direction of forward progression. KAM during the stance phase of gait (defined as foot contact to foot off a force plate) was calculated using inverse dynamics, using the local coordinate system of the femur. Knee Adduction Angular impulse is the area under the KAM curve, and was calculated using only positive KAM values (Bennell et al., 2011).

\subsection{Surgery}

Pre-operative planning is mandatory in osteotomy surgery (Elson et al., 2014) and this was performed to generate a simulated correction angle (Dugdale et al., 1992) and opening distance (Hernigou, 2002). Medial opening wedge HTO was performed (Lobenhoffer and Agneskirchner, 2003) to generate the planned opening distance before fixation with the TomoFix plate device (Depuy Synthes, Solothurn, Switzerland). Achieved correction was judged from post-operative long leg alignment radiographs by measuring the mechanical tibiofemoral angle (Paley et al., 1994) and the transection point of the weight bearing axis through the tibial plateau, expressed as a percentage of the tibial width from medial to lateral (Elson et al., 2014). Patients completed the Oxford knee score (Dawson et al., 1998).

\subsection{Statistics}

The average of the six walking trials was used for each participant, for toe out and natural gait. Student's $t$-test was performed to compare means from two groups. Analysis of variance (ANOVA) testing was performed where there were more than two groups, in which case post hoc tests were performed to identify significant differences between the groups. Levene's test was used to identify inequality of the variance, with Fishers least significant difference (LSD) post hoc test for groups of equal variance, and Games Howells post hoc test for groups of unequal variance. These were performed using SPSS v20.0 (IBM, Chicago, USA). Assumptions of normality were confirmed, and a $P$-value of 0.05 or less was considered a statistically significant result.

\section{Results}

\subsection{Participants}

The demographic details are outlined in Table 1 . The only statistically significant difference between the groups was age. Patients were diagnosed with medial compartment knee OA on the basis of history, clinical examination and plain radiographs, which were graded (Kellgren and Lawrence, 1957) for disease severity. There was a trend towards selecting younger patients for HTO (Price et al., 2013), and disease severity varied on an individual basis. Thirteen healthy controls were recruited from University staff and the student population. Height and body mass were not significantly different between the groups. Seventeen patients underwent

\section{Table 1}

Demographic data for each of the groups, values reported as means with standard deviation in brackets. Statistical testing with Student's t-test* and ANOVA**.

\begin{tabular}{lllll}
\hline Group & $\begin{array}{l}\text { Healthy } \\
\text { controls }\end{array}$ & $\begin{array}{l}\text { Pre-operative } \\
\text { patients }\end{array}$ & $\begin{array}{l}\text { Post-operative } \\
\text { patients }\end{array}$ & P-value \\
\hline $\mathrm{n}$ & 13 & 17 & 13 & \\
Age & $35.5(7.9)$ & $48.9(8.5)$ & $49.4(6.4)$ & $<0.001^{* *}$ \\
Height $(\mathrm{cm})$ & $171.2(9.7)$ & $173.9(11.0)$ & $173.1(11.7)$ & $\mathrm{ns}$ \\
Body mass $(\mathrm{kg})$ & $70.5(14.4)$ & $83.5(25.8)$ & $84.1(28.6)$ & $\mathrm{ns}$ \\
mTFA $\left(^{\circ}\right)$ & & $7.3(3.5)$ varus & $0.1(2.5)$ valgus & $<0.000^{*}$ \\
$\quad \begin{array}{l}\text { WBA } \% \text { tibial } \\
\quad \text { width }\end{array}$ & & $20.5(3.8)$ & $45.7(0.9)$ & $<0.000^{*}$ \\
$\begin{array}{l}\text { Oxford knee } \\
\quad \text { score }\end{array}$ & $26.1(8.2)$ & $39.6(5.4)$ & $<0.000^{*}$ \\
$\quad$ & & &
\end{tabular}


gait analysis prior to HTO surgery. The average age of the HTO patients was 48.9 years (SD 8.5 years) $(P<0.001), 15$ were male and 2 were female. 3 patients (17.6\%) had grade 2 arthritis, 12 (70.5\%) had grade 3 arthritis and 2 patients (11.7\%) presented with grade 4 arthritis. The mean mechanical tibio femoral angle was $7.3^{\circ}$ (SD 3.5 ) varus with the weight bearing axis crossing at $20.5 \%$ (SD 13.8\%) of the tibial width. Osteotomy surgery corrected the mean mechanical tibio femoral angle to $0.1^{\circ}$ (SD $2.5^{\circ}$ ) valgus with the weight bearing axis now crossing at $45.7 \%$ (SD $10.9 \%$ ) of the tibial width. Thirteen of these patients returned three to 24 months after surgery for further gait analysis. Clinical improvement was observed with an increase in the oxford knee score by 13.5 points $(P<0.001)$.

\subsection{Temporal parameters}

Healthy controls had a higher gait speed than the pre-operative groups (LSD $P<0.05$ ), Table 2 . There was no statistically significant difference between foot progression angles for TOG when performed preoperatively and post-operatively, and no difference for natural gait before and after surgery (Table 2). A significant difference was identified between healthy volunteers (Mean $14.06^{\circ}$ ) and natural gait (NG) groups (Mean $19.42^{\circ}$ ) for foot progression angle (LSD $P=0.032$ ).

\subsection{First peak KAM}

Pre-operative NG and TOG patients had a higher first peak KAM than post-op (Games Howells $P=0.004$ for NG and $P=0.009$ for TOG) and healthy controls (Games Howells $P=0.008$ for NG and $P=0.016$ for TOG) as charted in Table 2 . HTO reduced the mean first peak KAM by $45 \%$, to within normal limits (control range was $0.458-2.285 \%$ body weight $\times$ height $(\% \mathrm{BW} \cdot \mathrm{h}))$. TOG made no significant effect to first peak KAM, before or after HTO. First peak KAM values are summarised in Table 2.

\subsection{Second peak $K A M$}

Not all patients had a clear second peak KAM. Where no clear second peak KAM existed, the value of the KAM was taken which corresponded with the second peak of the ground reaction force, as described elsewhere (Guo et al., 2007; Hurwitz et al., 2002; Lynn et al., 2008; Shull et al., 2013). The highest second peak KAM achieved was from the preoperative natural gait group, the lowest was from the post-op TOG group as charted in Table 2. HTO reduced the second peak KAM (LSD $P=0.003$ ). TOG alone did not significantly reduce the second peak KAM, however when TOG was adopted after HTO the second peak KAM was reduced to below that of healthy controls (LSD $P=$ 0.021). Second peak KAM values are summarised in Table 2.

\subsection{Knee adduction angular impulse}

The KAAI was significantly reduced with HTO from $1.00 \% \mathrm{BW} \cdot \mathrm{h} \cdot \mathrm{s}$ $(\mathrm{SD}=0.54)$ to $0.52 \% \mathrm{BW} \cdot \mathrm{h} \cdot \mathrm{S}(\mathrm{SD}=0.29)(P=0.004)$, in line with healthy controls $0.59 \% \mathrm{BW} \cdot \mathrm{h} \cdot \mathrm{s}(\mathrm{SD}=0.22)$ (Table 2$)$. TOG did not significantly alter the KAAI in preoperative groups $(P=0.72)$, nor did it significantly alter the KAAI in the post-operative groups $(P=0.71)$ (Fig. 1).

\subsection{Sagittal plane data}

Knee flexion moment and angle are presented in Table 2. There was no significant change in peak knee flexion moment for preoperative patients with the addition of TOG $(P=0.794)$ and HTO $(P=0.229)$. TOG and HTO in isolation, however, significantly reduced knee flexion moment to below that of healthy controls. There was a small but insignificant increase in flexion moment post op, with the addition of TOG. The adoption of TOG reduced peak flexion angle to below healthy $(P=$ $0.002)$ and preoperative natural gait $(P=0.008)$ values, suggesting that this gait adaptation results in a smaller range of knee flexion.

\section{Discussion}

The effect of toe out gait on knee adduction moment has been studied previously. The first peak KAM is reported to be either increased (Bennell et al., 2011; Hurwitz et al., 2002; Lin et al., 2001; Lynn et al., 2008; van den Noort et al., 2013), is unchanged (Andrews et al., 1996; Caldwell et al., 2013; Guo et al., 2007; Lynn and Costigan, 2008; Schache et al., 2008; Shull et al., 2013) with adoption of TOG. It is

Table 2

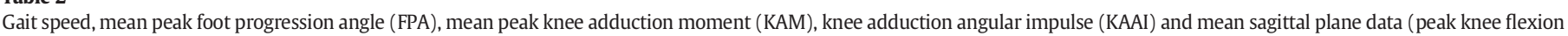

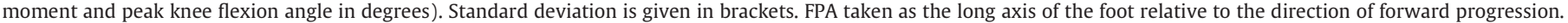
NG = natural gait, TOG $=$ toe out gait. The unit of KAM and peak flexion moment is $\%$ body weight $\times$ height and unit of KAAI is $\%$ body weight $\times$ height $\times$ seconds.

\begin{tabular}{|c|c|c|c|c|c|}
\hline \multirow{2}{*}{ Group } & \multirow{2}{*}{$\begin{array}{l}\text { Healthy controls } \\
\text { NG }\end{array}$} & \multicolumn{2}{|c|}{ Pre-operative patients } & \multicolumn{2}{|c|}{ Post-operative patients } \\
\hline & & NG & TOG & NG & TOG \\
\hline $\mathrm{FPA}\left({ }^{\circ}\right)^{*}$ & $14.06(3.78)$ & $19.42(6.59)$ & $30.77(6.93)$ & $19.77(7.12)$ & $32.25(8.03)$ \\
\hline Gait speed $(\mathrm{m} / \mathrm{s})^{* *}$ & $1.25(0.15)$ & $1.05(0.25)$ & $1.03(0.25)$ & $1.10(0.19)$ & $1.10(0.18)$ \\
\hline \multicolumn{6}{|c|}{$\begin{array}{l}\text { *Significance between healthy controls and pre/post-op NG }(P=0.032) \text { and pre/post-op TOG }(P=0.000) \text {. Significance between pre/post-op NG and pre/post-op TOG } \\
(P=0.000) \text {. } \\
{ }^{* *} \text { Significance between healthy controls and pre-op NG }(p=0.012) / \text { pre-op TOG }(P=0.006) \text {. }\end{array}$} \\
\hline First peak* & $1.59(0.54)$ & $2.70(1.06)$ & $2.85(1.36)$ & $1.51(0.50)$ & $1.88(0.60)$ \\
\hline Second peak ${ }^{* *}$ & $1.66(0.64)$ & $2.28(1.16)$ & $1.88(1.17)$ & $1.21(0.78)$ & $0.77(0.82)$ \\
\hline KAAI ${ }^{* * *}$ & $0.59(0.22)$ & $1.00(0.54)$ & $1.07(0.59)$ & $0.52(0.29)$ & $0.48(0.28)$ \\
\hline \multicolumn{6}{|c|}{$\begin{array}{l}\text { *Significance between healthy volunteers and pre-op NG }(P=0.008) / \text { pre-op TOG }(P=0.016) \text {. Significance between pre-op NG and post-op NG }(P=0.004) \text {. Significance } \\
\text { between pre-op TOG and post-op NG }(P=0.009) \text {. } \\
\text { ** Significance between healthy volunteers and post-op TOG }(P=0.021) \text {. Significance between pre-op NG and post-op NG }(P=0.003) / \text { post-op TOG }(P<0.001) \text {. Significance } \\
\text { between pre-op TOG and post-op TOG }(P=0.003) \text {. } \\
\text { *** Significance between pre-op NG and post-op NG }(P=0.004) / \text { post-op TOG }(P=0.002) / \text { healthy }(P=0.013) \text {. Significance between pre-op TOG and post-op NG } \\
(P=0.001) \text { /post-op TOG }(P<0.001) / \text { healthy }(P=0.004) \text {. }\end{array}$} \\
\hline Peak knee flexion moment* & $3.48(1.24)$ & $2.59(1.36)$ & $2.48(1.16)$ & $2.04(1.05)$ & $2.28(1.32)$ \\
\hline Knee flexion angle** & $62.15(3.16)$ & $61.27(3.39)$ & $57.85(4.42)$ & $61.39(4.36)$ & $58.3(2.37)$ \\
\hline
\end{tabular}




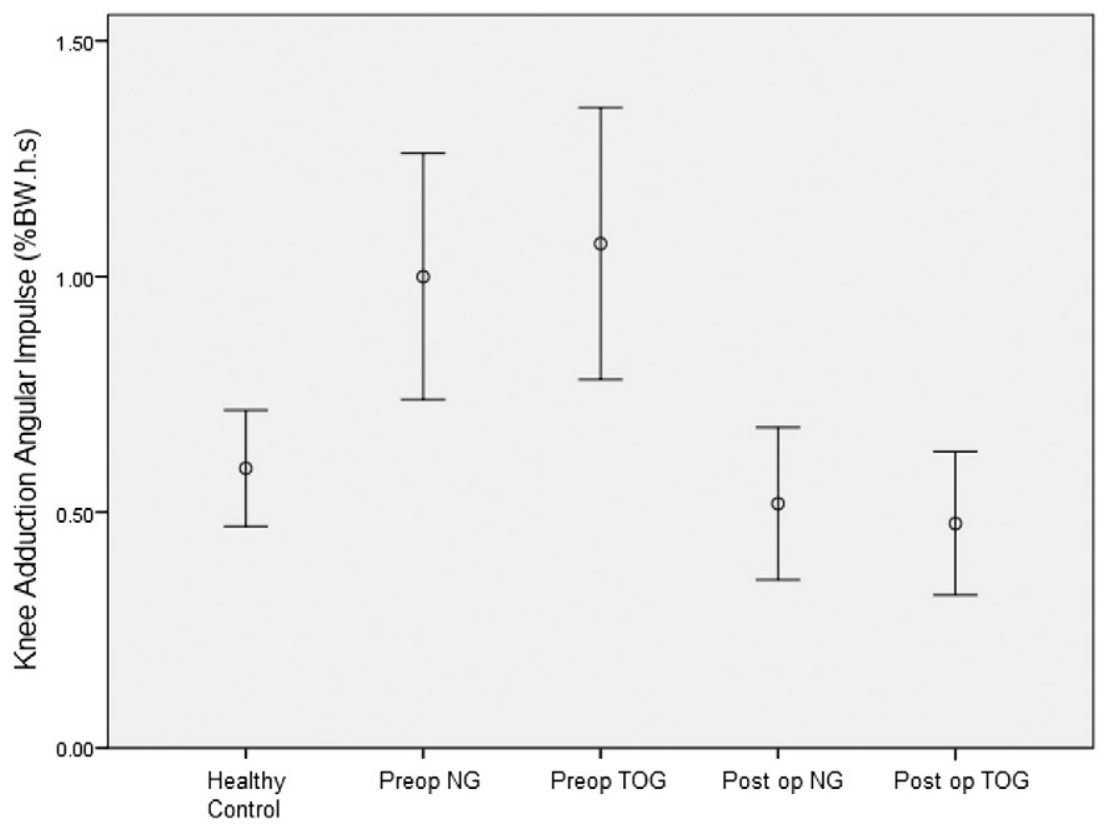

Fig. 1. Knee adduction angular impulse (KAAI) $(\% \mathrm{BW} \cdot \mathrm{h} \cdot \mathrm{s})$ for each cohort and gait style. Points represent means with whiskers representing plus or minus 2 standard errors.

generally reported that the second peak is lowered with the adoption of a TOG (Caldwell et al., 2013; Guo et al., 2007; Jenkyn et al., 2008; Lin et al., 2001; Lynn and Costigan, 2008; Lynn et al., 2008; Reinbolt et al., 2008; Schache et al., 2008; van den Noort et al., 2013).

Studies which have reported the results of KAM in \% body weight $\mathrm{x}$ height $(\% \mathrm{BW} \cdot \mathrm{h})$ are suggestive of first peak values between 2.70 and 3.78 and $2.84-4.70$ for natural gait and toe out respectively (Guo et al., 2007; Hunt and Takacs, 2014; Jenkyn et al., 2008; Simic et al., 2013; van den Noort et al., 2013). Second peak values are between 2.05 and 5.12 and $9.91-3.34 \% \mathrm{BW} \cdot \mathrm{h}$ for natural and toe out gait respectively (Guo et al., 2007; Hunt and Takacs, 2014; Jenkyn et al., 2008; Simic et al., 2013; van den Noort et al., 2013).

Toe out gait was investigated in this study because it has been extensively investigated previously, and is associated with slower disease progression (Chang et al., 2007), reduced knee pain (Hunt and Takacs, 2014), and it has been theorised as a self-adopted protective mechanism in OA (Chang et al., 2007), with benefits which extend post HTO (Wang et al., 1990). Toe in gait has been shown to increase the second peak and reduce the first peak KAM, however, toe out gait reduces second and increase first peak KAM, but also reduces KAM impulse (Simic et al., 2013), which is predictive of cartilage loss (Bennell et al., 2011), and better distinguishes disease severity (Kean et al., 2012).

In the literature, the first peak is reported to be clinically significant, correlating well with mechanical axis (Andrews et al., 1996; Hurwitz et al., 2002) and radiological predictors of severity (Hurwitz et al., 2002; Miyazaki et al., 2002). This study demonstrated a reduction in second peak KAM, but a small increase in the first peak with TOG for this patient group. Based on the increase in the first peak, and the current published literature, it is difficult to support the use of TOG to reduce medial compartment loading in patients with medial compartment $\mathrm{OA}$ and varus alignment. The reduction in the second peak with TOG in the pre-operative group is demonstrated in Table 2 , but this was not statistically significant.

Knee adduction angular impulse (sometimes referred to as KAM impulse) is a marker of dynamic medial joint load (Bennell et al., 2011) and associated with disease severity (Kean et al., 2012) and cartilage loss (Bennell et al., 2011). In this study, KAAI was not significantly altered by TOG, but was reduced by HTO. In a study by Hunt and Takacs (2014), KAAI was not significantly altered $(P=0.2)$ over 10 weeks, with a foot progression angle which was increased from $4.71^{\circ}$ to $11.41^{\circ}$ (Hunt and Takacs, 2014). Simic et al. (2013), also reported no significant reduction in KAAI with $10^{\circ}$ increases in target FPA, but significant reductions in second peak KAM and KAAI when target FPA went from $0^{\circ}$ to $30^{\circ}$ (Simic et al., 2013).

One theory to explain the results of TOG adaptation in this study, and other papers, is the magnitude of change in FPA from patients self-selected FPA. In this study, the natural FPA of patients with medial compartment $\mathrm{OA}\left(19.42^{\circ}, \mathrm{SD} 6.59^{\circ}\right)$ was greater than that of the healthy controls $\left(14.06^{\circ}, \mathrm{SD} 3.78^{\circ}\right.$ ) (Table 2 ), which could reflect the subtle use of TOG as a subconscious compensatory mechanism as speculated by Wang et al. (1990). Comparing the FPA results in this study with those published previously (Hunt and Takacs, 2014; Simic et al., 2013), the population study in this report had a naturally high FPA. It is less likely, therefore, to find significant improvements in second peak KAM or KAAI by increasing FPA from 20 to $30^{\circ}$. Similarly, Simic et al. (2013) reported no significant difference in peak KAM or KAAI between $20^{\circ}$ target (FPA average $12.6^{\circ}$ ) and $30^{\circ}$ target (FPA average $20.8^{\circ}$ ) (Simic et al., 2013). The data from this study, and the study by Simic et al. (2013), suggest that any benefit of TOG on medial load is dependent on the achievable increase in FPA with TOG, related to the patients preexisting natural FPA.

The biomechanical benefit of HTO on both peaks of the KAM, and KAAI, is clear and has been previously reported (Bhatnagar and Jenkyn, 2010; Birmingham et al., 2009; Briem et al., 2007; Lind et al., 2013; Prodromos et al., 1985). It has been reported that the mechanical axis (limb alignment) was the single best predictor of both KAM peaks (Hurwitz et al., 2002). Preoperative patients had a raised first $(P<0.05)$ and second peak KAM (non-significant) compared to healthy controls, this was then reduced after surgery, to a level not significantly different to that of the healthy group, the age of which was significantly lower than the operative groups $(P<0.001)$. HTO surgery significantly reduced the first peak, second peak KAM and KAAI, when walking with a natural FPA $(P=0.004, P=0.003, P=0.004)$.

Adopting TOG after HTO appears to lower the second peak KAM further below that of control levels $(P=0.021)$, with an associated but not statistically significant increase in first peak KAM. The effect on KAAI is not significantly different. The implications of this are unclear, but may represent further offloading of the medial compartment, possibly increasing the load to the lateral compartment. This reduction in the 2nd peak KAM would be expected to slow disease progression in the affected medial compartment, potentially prolonging the beneficial effects of surgery, but the risk of this causing disease progression in the 
lateral compartment is not known. Further longitudinal studies would be required to test any such assertion.

Prodromos et al. (1985) and Wang et al. (1990) reported favourable long term outcomes in those with low KAM pre-operatively compared to high KAM pre-operatively, citing TOG as an important predictor of KAM. The adoption of TOG could enhance the longevity of alteration in KAM achieved after HTO, with potential clinical benefits.

Data for knee flexion angle and sagittal plane moment is also presented for completeness. Neither of the interventions, TOG, HTO or a combination, returned knee flexion moment to healthy levels. Simulated results from a previous study had suggested that this would be increased as the medial knee compartment was off loaded (Jenkyn et al., 2008). A more recent study, however, by Simic et al. (2013), reported reductions in knee flexion moment with TOG, and increases in first peak KAM (Simic et al., 2013). The peak knee flexion values were reduced with toe out gait.

This is the first study to compare the effects of TOG on pre- and postoperative HTO patients. In a population with a higher foot progression angle, TOG has no significant effects on first peak, second KAM peak or KAAI. The findings highlight the biomechanical advantages of HTO in returning first and second peak KAM to within healthy limits. The aim of this study was to assess TOG as an alternative to surgery or an addition to surgery by its effect on KAM. The results show that TOG does not alter the KAM in isolation, when compared to anatomical correction of the mechanical axis, as in HTO, and is not a viable alternative to surgical management in such patients. The adoption of TOG post-operatively reduces second peak KAM to below that of healthy controls, but this is not evidence enough to advocate this addition as excessive loading of the lateral compartment is a concern.

Limitations of this study are a non-aged matched volunteer healthy group and small cohort sizes. Although the mean age of the control group is lower than the patient cohorts, this study shows that following HTO, biomechanical measures of knee loading are improved to similar values as the younger control group, adding weight to the conclusion. A modest number of patients were assessed and this is in line with other similar studies. The investigation of just one gait modification, TOG, and the lack of longitudinal results for retention and effectiveness may also be viewed as limitations. However, with the results of this preliminary study which considers TOG with HTO patients for the first time, it would be difficult to recommend long term adoption of TOG in this patient population.

\section{Conclusion}

This study compares the individual and combined effects of TOG and HTO on KAM in a clinically defined patient population. TOG does not significantly reduce first peak, second peak KAM or KAAI in patients with medial compartment OA and varus alignment deformity. This could be due to a higher self-selected FPA in the population of this study, and is suggestive that any benefit of TOG may be dependent on the achievable increase in FPA with TOG, related to the patients pre-existing natural FPA. HTO however, results in clear reductions in both peaks, restoring them to within healthy limits. One particularly interesting finding is that HTO with TOG reduces the second KAM peak to below that of healthy controls. This requires further study to define the clinical implications, particularly regarding HTO longevity. Results for the sagittal plane demonstrated reductions in peak knee flexion angle with TOG, and that TOG or HTO did not return knee flexion moment to normal. The potential of other gait adaptation techniques to further reduce KAM after surgery, as well as the distribution of forces and moments to other structures, are topics for further research.

\section{Acknowledgement}

This work was supported by Arthritis Research UK [grant number 18461], EPSRC [EP/J010111/1] and NISCHR CRC.

\section{References}

Amis, A.A., 2013. Biomechanics of high tibial osteotomy. Knee Surg. Sports Traumatol. Arthrosc. 21, 197-205

Andrews, M., Noyes, F.R., Hewett, T.E., Andriacchi, T.P., 1996. Lower limb alignment and foot angle are related to stance phase knee adduction in normal subjects: a critical analysis of the reliability of gait analysis data. J. Orthop. Res. 14 (2), 289-295.

Bennell, K.L., Bowles, K.A., Wang, Y., Cicuttini, F., Hinman, T., 2011. Higher dynamic medial knee load predicts greater cartilage loss over 12 months in medial knee osteoarthritis. Ann. Rheum. Dis. 70, 1770-1774.

Bhatnagar, T., Jenkyn, T.R., 2010. Internal kinetic changes in the knee due to high tibial osteotomy are well correlated with change in external adduction moment: an osteoarthritic knee model. J. Biomech. 43, 2261-2266.

Birmingham, T.B., Giffin, J.R., Chesworth, B.M., Bryant, D.M., Litchfield, R.B., Willits, K., Jenkyn, Chris T.R., Fowler, P.J., 2009. Medial opening wedge high tibial osteotomy: a prospective cohort study of gait, radiographic, and patient-reported outcomes. Arthritis Rheum. 61 (5), 648-657.

Briem, K., Ramsey, D.K., Newcomb, W., Rudolph, K.S., Snyder-Mackler, L., 2007. Effects of the amount of valgus correction for medial compartment knee osteoarthritis on clinical outcome, knee kinetics and muscle co-contraction after opening wedge high tibial osteotomy. J. Orthop. Res. 25 (3), 311-318.

Caldwell, L.k., Laubach, L.L., Barrios, J.A., 2013. Effect of specific gait modifications on medial knee loading, metabolic cost and perception of task difficulty. Clin. Biomech. 28 (6), 649-654.

Chang, A., Hurwitz, D., Dunlop, D., Song, J., Cahue, S., Hayes, K., 2007. The relationship between toe-out angle during gait and progression of medial tibiofemoral osteoarthritis. Ann. Rheum. Dis. 66, 1271-1275.

Dawson, J., Fitzpatrick, R., Murray, D., Carr, A., 1998. Questionnaire on the perceptions of patients about total knee replacement. J. Bone Joint Surg. (Br.) 80-B, 63-69.

Dugdale, T.W., Noyes, F.R., Styer, D., 1992. Preoperative planning for high tibial osteotomy. The effect of lateral tibiofemoral separation and tibiofemoral length. Clin. Orthop. Relat. Res. 274, 248-264

Elson, D.W., Petheram, T.G., Dawson, M.J., 2014. High reliability in digital planning of medial opening wedge high tibial osteotomy, using Miniaci's method. Knee Surg. Sports Traumatol. Arthrosc. (Available as ePub). 10.1007/s00167-014-2920-x.

Guo, M., Axe, M.J., Manal, K., 2007. The influence of foot progression angle on the knee adduction moment during walking and stair climbing in pain free individuals with knee osteoarthritis. Gait Posture 26, 436-441.

Hernigou, P., 2002. Open wedge tibial osteotomy: combined coronal and sagittal correction. Knee 9, 15-20.

Hunt, M., Bennell, K., 2011. Predicting dynamic knee joint load with clinical measures in people with medial knee osteoarthritis. Knee 18 (4), 231-234.

Hunt, M.A., Takacs, J., 2014. Effects of a 10-week toe-out gait modification intervention in people with medial knee osteoarthritis: a pilot, feasibility study. Osteoarthr. Cartil. 22, 904-911.

Hurwitz, D.E., Ryals, A.B., Case, J.P., Block, J.A., Andriacchi, T.P., 2002. The knee adduction moment during gait in subjects with knee osteoarthritis is more closely correlated with static alignment than radiographic disease severity, toe out angle and pain. J. Orthop. Res. 20, 101-107.

Jenkyn, T.R., Hunt, M.A., Jones, I.C., Giffin, J.R., Birmingham, T.B., 2008. Toe-out gait in patients with knee osteoarthritis partially transforms the external knee adduction moment into flexion moment during early stance phase of gait: a tri-planar kinetic mechanism. J. Biomech. 41, 276-283.

Kean, C.O., Hinman, R.S., Bowles, K.A., Cicuttini, F., Davies-Tuck, M., Bennell, K.L., 2012. Comparison of peak knee adduction moment and knee adduction moment impulse in distinguishing between severities of knee osteoarthritis. Clin. Biochem. 27, 520-523.

Kellgren, J.H., Lawrence, J.S., 1957. Radiological assessment of osteo-arthrosis. Ann. Rheum. Dis. 16 (4), 494-502.

Lin, C.J., Lai, K.A., Chou, Y.L., Ho, C.S., 2001. The effect of changing the foot progression angle on the knee adduction moment in normal teenagers. Gait Posture 14, 85-91.

Lind, M., McClelland, J., Wittwer, J.E., Whitehead, T.E., Feller, J.A., Webster, K.E., 2013. Gait analysis of walking before and after medial opening wedge high tibial osteotomy. Knee Surg. Sports Traumatol. Arthrosc. 21, 74-81.

Lobenhoffer, P., Agneskirchner, J.D., 2003. Improvements in surgical technique of valgus high tibial osteotomy. Knee Surg. Sports Traumatol. Arthrosc. 11 (3), $132-138$.

Lynn, S.K., Costigan, P.A., 2008. Effect of foot rotation on knee kinetics and hamstring activation in older adults with and without signs of knee osteoarthritis. Clin. Biomech. 23, 779-786.

Lynn, S.K., Kajaks, T., Costigan, P.A., 2008. The effect of internal and external foot rotation on the adduction moment and lateral-medial shear force at the knee during gait. J. Sci. Med. Sport 11, 444-451.

Miyazaki, T., Wada, M., Kawahara, H., Sato, M., Baba, H., Shimada, S., 2002. Dynamic load at baseline can predict radiographic disease progression in medial compartment knee steoarthritis. Ann. Rheum. Dis. 61, 617-622.

Mundermann, A., Dyrby, C.O., Andriacchi, T.P., 2005. Secondary gait changes in patients with medial compartment knee osteoarthritis increased load at the ankle, knee, and hip during walking. Arthritis Rheum. 52 (9), 2835-2844.

Paley, D., Herzenberg, J.E., Tetsworth, K., McKie, J., Bhave, A., 1994. Deformity planning for frontal and sagittal plane corrective osteotomies. Orthop. Clin. North Am. 25 (3), 425-465.

Price, A., Beard, D., Thienpont, E., 2013. Uncertainties surrounding the choice of surgical treatment for 'bone on bone' medial compartment arthritis of the knee. Knee 20 (S1), S16-S20. 
Prodromos, C.C., Andriacchi, T.P., Galante, J.O., 1985. A relationship between gait and clinical changes following high tibial osteotomy. J. Bone Joint Surg. Am. 67 (8), 1188-1194.

Reinbolt, J.A., Haftka, R.T., Hmielewski, T.L., Fregly, B.J., 2008. A computational framework to predict post-treatment outcome for gait-related disorders. Med. Eng. Phys. 30 (4), 434-443.

Rutherford, D.J., Hubley-Kozey, C.L., Deluzio, K.J., Stanish, W.D., Dunbar, M., 2008. Foot progression angle and the knee adduction moment: a cross-sectional investigation in knee osteoarthritis. Osteoarthr. Cartil. 16, 883-889.

Schache, A.G., Fregly, B.J., Crossley, K.M., Hinman, R.S., Pandy, M.G., 2008. The effect of gait modification on the external knee adduction moment is reference frame dependent. Clin. Biomech. 23, 601-608.

Shull, P.B., Shultz, R., Silder, A., Dragoo, J.L., Besier, T.F., Cutkosky, M.R., Delp, S.L., 2013. Toe-in gait reduces the first peak knee adduction moment in patients with medial compartment knee osteoarthritis. J. Biomech. 46, 122-128.

Simic, M., Hinman, R.S., Wrigley, T.V., Bennell, K.L., Hunt, M.A., 2011. Gait modification strategies for altering medial knee joint load: a systematic review. Arthritis Care Res. 63 (3), 405-426.

Simic, M., Wrigley, T.V., Hinman, R.S., Hunt, M.A., Bennell, K.L., 2013. Altering foot progression angle in people with medial knee osteoarthritis: the effects of varying toe-in and toe-out angles are mediated by pain and malalignment. Osteoarthr. Cartil. 21, $1272-1280$.
Thorp, L.E., Wimmer, M.A., Block, J.A., Moisio, K.C., Shott, S., Goker, B., Sumner, D.R., 2006 Bone mineral density in the proximal tibia varies as a function of static alignment and knee adduction angular momentum in individuals with medial knee osteoarthritis. Bone 39, 1116-1122.

Thorp, L.E., Sumner, D.R., Wimmer, M.A., Block, J.A., 2007. Relationship between pain and medial knee joint loading in mild radiographic knee osteoarthritis. Arthritis Rheum. 57 (7), 1254-1260.

van den Noort, J.C., Schaffers, I., Snijders, J., Harlaar, J., 2013. The effectiveness of voluntary modifications of gait pattern to reduce the knee adduction moment. Hum. Mov. Sci. $32,412-424$.

Walter, J.P., D'Lima, D.D., Colwell, C.W., Fregly, B.J., 2010. Decreased knee adduction moment does not guarantee decreased medial contact force during gait. J. Orthop. Res. 28 (10), 1348-1354.

Wang, J.W., Kuo, K.N., Andriacchi, T.P., Galante, J.O., 1990. The influence of walking mechanics and time on the results of proximal tibial osteotomy. JBJS 6 (72A), 905-909.

Weidenhielm, L., Svensson, O.K., Broström, L.Å., 1992. Change of adduction moment about the hip, knee and ankle joints after high tibial osteotomy in osteoarthrosis of the knee. Clin. Biomech. 7 (3), 177-180.

Zhao, D., Banks, S.A., Mitchell, K.H., D'Lima, D.D., Colwell, C.W., Fregly, B.J., 2007. Correlation between the knee adduction torque and medial contact force for a variety of gait patterns. J. Orthop. Res. 25 (6), 789-797. 